\title{
Visualization of Photoinduced Charge Transfer and Electron-Hole Coherence in Two-Photon Absorption
}

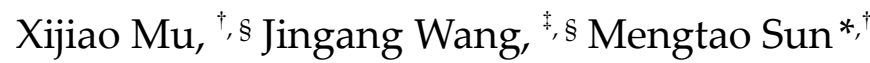

† School of Mathematics and Physics, Beijing Key Laboratory for Magneto-Photoelectrical Composite and Interface Science, University of Science and Technology Beijing, Beijing, 100083, P.R. China.

${ }^{\ddagger}$ College of Science, Liaoning Shihua University, Fushun, 113001, PR China

* Corresponding author. Email: mengtaosun@ustb.edu.cn (M. Sun).

$\S$ X.M. and J.W. contributed equally.

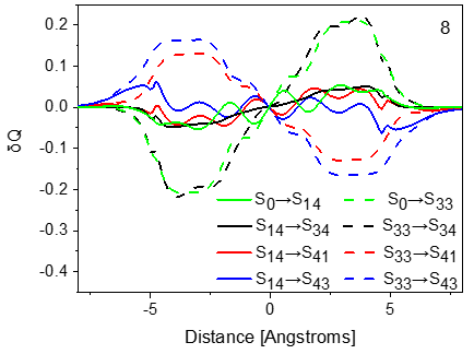

(a)

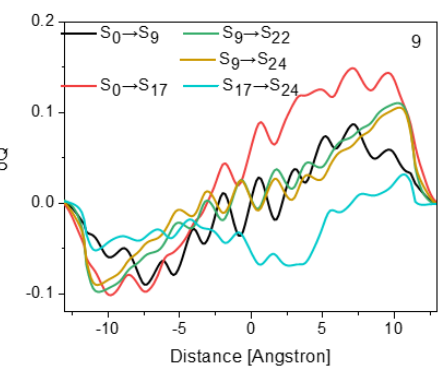

(b)

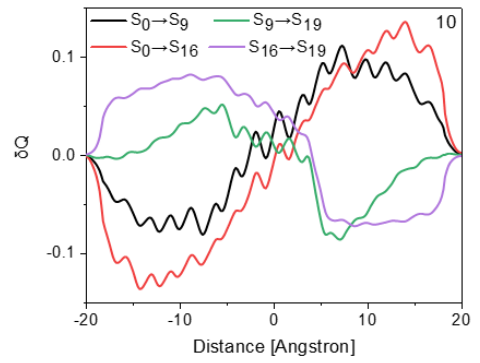

(c)

Figure S1. The charge transfer integral curve of three molecules. Molecules 8 (a), molecules 9 (b) and molecules 10 (c). 